\title{
Response to "Commentary on: Stretching is Superior to Brisk Walking for Reducing Blood Pressure in People With High-Normal Blood Pressure or Stage I Hypertension"
}

\author{
Philip D. Chilibeck
}

Thank you for the opportunity to respond to the questions in the commentary ${ }^{1}$ about our recent study. The discrepancy between the manuscript and the trial registration is due to an error on the trial registration number mentioned in the manuscript. The registration number should be NCT03947996 instead of NCT02391779. We have requested the journal publish an erratum to correct this error.

As mentioned in the commentary, ${ }^{1}$ our previous study involving flax lignan supplementation and a walking program (NCT02391779) had the goal of recruiting 100 participants. Our hypothesis in that study was that combining flax lignan and a walking intervention would be additive for decreasing blood pressure. Due to stringent inclusion/exclusion criteria, which restricted the study to participants $\geq 60$ years, we were only able to recruit 25 participants and the study was closed in July 2017. Neither the flax supplement nor the walking program had effects on blood pressure, and the results were published as a conference abstract. ${ }^{2}$ We noticed from this study that our placebo-exercise group (which was assigned a stretching program) unexpectedly had trends for reducing blood pressure. This motivated a search of stretching studies from the literature and identification of other studies that found benefits of stretching for vascular function and reducing blood pressure. . $^{3,4}$ These studies led to the hypothesis that we proposed in our recent publication that stretching would be superior to a walking program for reducing blood pressure. ${ }^{5}$ For this study, we opened our recruitment to participants $\geq 18$ years with high-normal or stage I hypertension to increase participant numbers. These participants were run through an additional study from May to September 2019, which was registered separately (NCT03947996) and involved randomization of the participants to walking or stretching groups, with the outcomes including daytime, nighttime, sitting, and lying blood pressure measurements mentioned in our trial registry.
The issue of including multiple outcomes analyzed in our trial is a limitation regarding the inflation of type I statistical error. As mentioned in our manuscript, our study was limited by a small sample size; therefore, it was felt that there was a lack of statistical power to correct our alpha level for multiple outcome measurements. It is recognized that our findings need to be replicated in a larger clinical trial to overcome this limitation.

\section{References}

1. Hansford HJ, Wewege MA, Jones MD. Commentary on: Stretching is superior to brisk walking for reducing blood pressure in people with high-normal blood pressure or stage 1 hypertension. J Phys Act Health. Published online March 10, 2021. doi:10.1123/jpah.2021-0049

2. Ko J, Chilibeck P, Alcorn J, Hadjistavropoulos T. Effect of flax lignan combined with exercise training on blood pressure of older adults with prehypertension or stage I hypertension. Appl Physiol Nutr Metab. 2017;42(suppl 2):S82-S83.

3. Shinno H, Kurose S, Yamanaka Y, et al. Evaluation of a static stretching intervention on vascular endothelial function and arterial stiffness. Eur J Sport Sci. 2017;17(5):586-592. PubMed ID: 28287021 doi:10.1080/17461391.2017.1284267

4. Wong A, Figueroa A. Eight weeks of stretching training reduces aortic wave reflection magnitude and blood pressure in obese postmenopausal women. J Hum Hypertens. 2014;28(4):246-250. PubMed ID: 24132138 doi:10.1038/jhh.2013.98

5. Ko J, Deprez D, Shaw K, et al. Stretching is superior to brisk walking for reducing blood pressure in people with high-normal blood pressure or stage I hypertension. J Phys Act Health. 2021;18(1): 21-28. doi:10.1123/jpah.2020-0365 